\title{
Diseño de experimentos para optimizar resistencia e índices de capacidad de un fusible
}

\section{Design of experiments to optimize fuse resistance and capacity indices}

Jesús Antonio Torres-Sosa ${ }^{1}$, Luis Alberto Rodríguez-Picón ${ }^{1}$, Luis Carlos Méndez-González1 , Iván Juan Carlos Pérez-Olguín'

1 Universidad Autónoma de Ciudad Juárez

\section{RESUMEN}

Las empresas de manufactura buscan ofrecer productos de calidad a sus clientes. Sin embargo, la calidad es subjetiva, en función de los requerimientos únicos de cada producto y cómo estos son afectados por distintas condiciones a los que está sometido el material durante su proceso de fabricación. Por ello, se busca, por medio de estudios de capacidad de proceso, verificar que las especificaciones de los productos cumplan con lo solicitado por el cliente. La resistencia en un fusible evita que los equipos sufran sobrecargas de energía, actuando como un dispositivo de seguridad y, debido a ello, es importante que la resistencia cumpla con su función y evite daños graves. Por medio del análisis de índices de capacidad, es factible concluir si un proceso es capaz o no, evaluando una característica crítica del producto. Cuando los índices muestran un proceso no capaz, se busca, con distintas herramientas estadísticas, encontrar una solución para evitar deficiencias en el proceso. En el presente artículo se reporta el desarrollo de un diseño de experimentos para optimizar la resistencia en un fusible y mejorar los índices de capacidad del proceso. Se presenta un esquema de experimentación para la optimización de los parámetros del proceso y al final se logró incrementar la capacidad del proceso, pasando de un Cpk de 0.25 a 1.24, lo cual denota la mejora del mismo.

PALABRAS CLAVE: Diseño de experimentos; índices de capacidad; resistencia de un fusible.

\section{ABSTRACT}

Manufacturing companies seek to offer quality products to their customers. However, quality is subjective depending on the unique requirements of each product and how these are affected by different conditions that the material is subjected to during its manufacturing process. Therefore, it is sought by means of process capacity studies to verify that the specifications of the products comply with what is requested by the client. The resistance in a fuse prevents equipment from undergoing power surges, acting as a safety device and, because of this, it is important that the resistance fulfills its function and prevents serious damage. Through the analysis of capacity indices, it is possible to conclude whether a process is capable or not, evaluating a critical characteristic of the product. When the indices show a non-capable process, different statistical tools are used to find a solution to avoid deficiencies in the process. In this article, a design of experiments is developed to optimize resistance in a fuse and improve the capacity indices of the process. An experimentation scheme is presented for the optimization of the process' parameters and after the experimentation it was possible to increase the process capability going from a $0.25 \mathrm{Cpk}$ level to a 1.24 Cpk level, which denotes the improvement of the process.

KEYWORDS: Design of experiments; capacity indices; fuse resistance.

Correspondencia:

DESTINATARIO: Luis Alberto Rodríguez Picón INSTITUCIÓN: Universidad Autónoma de Ciudad Juárez DIRECCIÓN: Av. del Charro núm. 450 norte, col. Partido Romero, C. P. 32310, Ciudad Juárez, Chih., México CORREO ELECTRÓNICO: luis.picon@uacj.mx
Fecha de recepción: 11 de noviembre de 2020. Fecha de aceptación: 21 de diciembre de 2020. Fecha de publicación: 26 de diciembre de 2020.
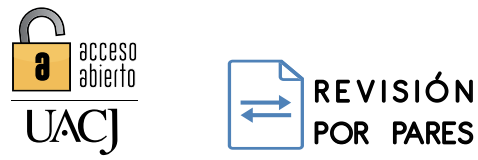

Licencia Creative Commons

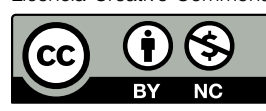




\section{INTRODUCCIÓN}

Desde el punto de vista de los clientes, las empresas existen para proveer un bien o un servicio, ya que ellos necesitan productos con características que cubran ciertas necesidades [1]. Todo producto pasa por un proceso, el cual transforma la materia prima e insumos en productos terminados o partes de un ensamble mayor.

De acuerdo con [2], un proceso de manufactura está sometido a distintos factores de carácter aleatorio que hacen imposible fabricar productos exactamente iguales. Se han desarrollado herramientas estadísticas para estudiar estos factores y evaluar que los procesos se encuentren controlados. Uno de los principales instrumentos para realizar un análisis y determinar si el proceso es apto para cumplir con los requerimientos del cliente son los estudios de capacidad del proceso, los cuales normalmente validan una sola variable.

La variación en las características de los productos es inevitable, por lo cual se cuenta con un rango de tolerancia que permite mantener una correcta fabricación de los productos.

Un estudio de capacidad de proceso es una investigación planificada minuciosamente, de modo que genere información específica sobre el rendimiento de un proceso en condiciones determinadas de operación [3].

Como plantea ${ }^{[4]}$, en el campo de la industria es frecuente realizar experimentos con la finalidad de resolver un problema o comprobar alguna idea, por ejemplo, se hacen cambios en los insumos, métodos o condiciones de un proceso, cambiando la presión de una máquina para estimar mejoras o eliminar defectos.

Se puede suponer que para la industria, la calidad es sumamente importante y, por ello, es necesario conocer, mejorar y mantener los procesos para asegurarla.

\section{Índices de capacidad univariados}

Los índices de capacidad fueron creados para cumplir adecuadamente con la función de predecir cuántos de los productos del proceso van a satisfacer a las especificaciones ${ }^{[5]}$. Los procesos tienen variables de salida o de respuesta, las cuales deben cumplir con ciertas especificaciones a fin de considerar que el proceso está funcionando de manera satisfactoria ${ }^{[6]}$. Evaluar la habilidad o capacidad de un proceso consiste en conocer el alcance de la variación natural de este para una característica de calidad dada, lo cual permitirá conocer en qué medida tal característica de calidad cumple sus especificaciones ${ }^{[1]}$. La forma común de expresar la capacidad es en términos de índices o medidas adimensionales que cuantifican el comportamiento del proceso, teniendo en cuenta los parámetros del mismo y las especificaciones del producto [7]. Los estudios de capacidad tienen los índices $C p$ y $C p k$, que son utilizados para evaluar la capacidad del proceso.

Asimismo [1], expresan que el índice de capacidad potencial del proceso $(C p)$ se define como se muestra en la Ecuación (1):

$$
C p=\frac{E S-E I}{6 \sigma}
$$

donde $\sigma$ representa la desviación estándar del proceso, mientras que $E S$ y $E I$ son las especificaciones superior e inferior para la característica de calidad. Como se puede observar, el índice $C p$ compara el ancho de las especificaciones o la variación tolerada para el proceso con la amplitud de la variación real de este, por lo que se puede definir como:

$$
C p=\frac{\text { Variación tolerada }}{\text { Variación real }}
$$

De acuerdo a [1], en la Tabla 1 se muestran los valores y una breve descripción de cómo pueden ser descritos, según los resultados obtenidos.

TABLA 1

VALORES de $C P$ y SU INTERPRETACión [1]

\begin{tabular}{|l|l|l|}
\hline $\begin{array}{l}\text { VALOR DEL } \\
\text { ÍNDICE Cp }\end{array}$ & $\begin{array}{c}\text { CLASE O } \\
\text { CATEGORÍA } \\
\text { DEL } \\
\text { PROCESO }\end{array}$ & \multicolumn{1}{|c|}{ DeCisión } \\
\hline$C p \geq 2$ & $\begin{array}{l}\text { Clase } \\
\text { mundial }\end{array}$ & Se tiene calidad Seis Sigma. \\
\hline$C p>1.33$ & 1 & Adecuado. \\
\hline $1<C p<1.33$ & 2 & $\begin{array}{l}\text { Parcialmente adecuado, requiere de } \\
\text { un control estricto. }\end{array}$ \\
\hline $0.67<C p<1$ & 3 & $\begin{array}{l}\text { No adecuado para el trabajo. Es ne- } \\
\text { cesario un análisis del proceso. Re- } \\
\text { quiere de modificaciones serias para } \\
\text { alcanzar una calidad satisfactoria. }\end{array}$ \\
\hline$C p<0.67$ & 4 & $\begin{array}{l}\text { No adecuado para el trabajo. } \\
\text { Requiere de modificaciones serias. }\end{array}$ \\
\hline
\end{tabular}


Con base en [1], el índice Cpk es también conocido como índice de capacidad real del proceso y es considerado una versión corregida del $C p$ que sí toma en cuenta el centrado del proceso. Existen varias formas equivalentes para calcularlo, una de las más comunes es la Ecuación (3):

$$
C p k=\text { Mínimo } x\left[\frac{\mu-E I}{3 \sigma}, \frac{E S-\mu}{3 \sigma}\right]
$$

Como se aprecia, el índice $C p k$ es igual al valor más pequeño de entre $C p i$ y $C p s$, es decir, es igual al índice unilateral más pequeño, por lo que si el valor de $C p k$ es satisfactorio (mayor que 1.25), eso indica que el proceso en realidad es capaz. Si $C p k<1$, entonces el proceso no cumple con por lo menos una de las especificaciones. Algunos elementos adicionales para la interpretación del índice $C p k$ son los siguientes:

- El índice Cpk siempre va a ser menor o igual que el índice $C p$. Cuando son muy próximos, eso indica que la media del proceso está muy cerca del punto medio de las especificaciones, por lo que la capacidad potencial y real son similares.

- Si el valor del índice $C p k$ es más pequeño que el $C p$, esto indica que la media del proceso está alejada del centro de las especificaciones ${ }^{[7]}$.

\section{Diseño de experimentos}

El diseño factorial considera todas las posibles combinaciones de variables, lo que lleva a muchas condiciones experimentales ${ }^{[8]}$. Considere un diseño factorial de $2^{k}$ que consiste en $N=2^{k}$ tratamientos formados a partir de todas las combinaciones posibles de los dos niveles de todos los $k$ factores. Los dos niveles de cualquier factor generalmente se denominan nivel inferior y nivel superior de ese factor bajo el control del experimentador. Por ejemplo, si uno de los factores es la temperatura, entonces los dos niveles podrían ser las temperaturas superior e inferior de $100{ }^{\circ} \mathrm{C}$ y $120^{\circ} \mathrm{C}$. Estos diseños se denominan con frecuencia factoriales de dos niveles, sin tener en cuenta el valor cualitativo o naturaleza cuantitativa de los factores controlados ${ }^{[9]}$.

Como hace referencia [10], cuando existe variación en el proceso, el diseño de experimentos es una técnica estadística utilizada para determinar el ajuste óptimo de los factores que influyen al proceso y, de esta manera, perfeccionar su funcionamiento, reducir su variabilidad y mejorar la manufactura de productos. De acuerdo con [11], los diseños factoriales son más eficientes que los unifactoriales debido a que se puede analizar no solo los impactos individuales sino también los efectos combinados de factores.

En el caso en que el número de factores descrito por $k$ sea elevado, el número de tratamientos requeridos por los diseños factoriales de $2^{k}$ se vuelven aun más grandes. Por ejemplo, una réplica completa del diseño 27 requiere 128 ciclos de tratamiento. Cuando se completan, los datos de un diseño $2^{k}$ proporcionan, además de las estimaciones de los $k$ efectos principales y las $k(k-1) / 2$ interacciones de dos factores, estimaciones de todos los efectos de tres factores y de orden superior. Sin embargo, a menudo se da el caso de que se puede suponer a priori que los efectos de tres factores y de orden superior son cero o al menos pequeños en relación con los efectos de orden inferior. Cuando esto es cierto, solo es necesario emplear una fracción del diseño $2^{k[9]}$.

\section{Diseño factorial fraccionado $2^{k-1}$}

La notación $2^{k-1}$ significa una fracción del diseño factorial completo $2^{k}$ que tiene sentido fraccionar cuando $k$ es mayor a 2 [9]. Los diseños factoriales fraccionados, como su nombre lo indica, son solamente una parte de los diseños factoriales completos, que permiten reducir el número de corridas experimentales [12].

Los diseños factoriales fraccionados se utilizan como una opción cuando los recursos que se tienen son pocos o el número de factores incluidos en el diseño es grande, porque la información que presenta incluye menor cantidad de corridas que el diseño factorial completo. Cabe mencionar que al presuponer que hay factores que se pueden descartar al fraccionar el diseño completo, implica una pérdida de información.

En la práctica, en lugar de crear un diseño factorial completo y eliminar factores hasta obtener la fracción deseada, lo que se hace es crear un diseño completo con el número de corridas elegidas y se agregan los factores adicionales al diseño ${ }^{[13]}$.

\section{METODOLOGÍA}

La implementación del diseño de experimentos se realizó de manera secuencial. Antes de iniciar con el experimento, se aseguró que los instrumentos de medición 
tuvieran calibración vigente, ya que esto contribuye a la calidad de los datos y la reducción de errores.

En la Figura 1 se muestra la metodología utilizada para la implementación del diseño de experimentos.

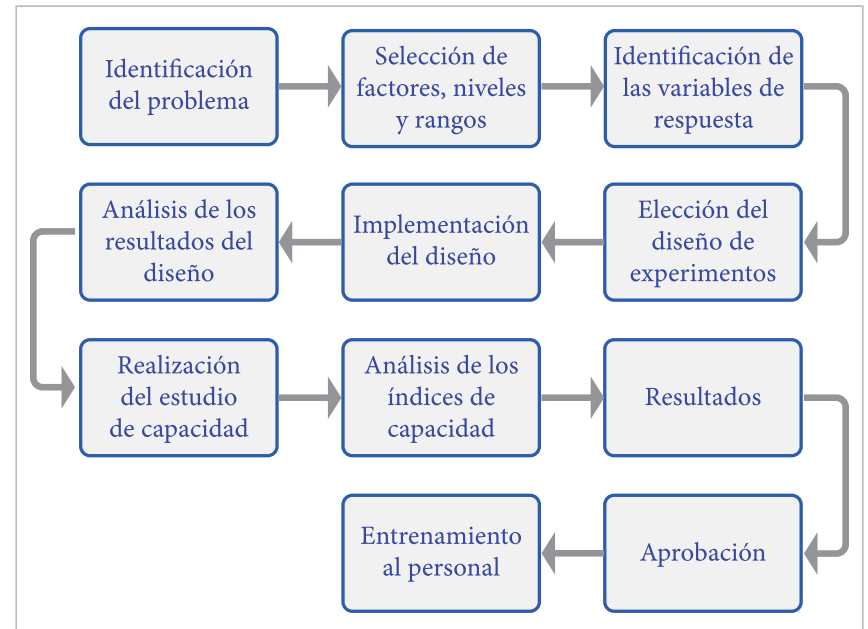

Figura 1. Diagrama de bloques de la metodología [4].

Para verificar la correcta construcción del fusible se hizo una corrida de validación piloto, tomando en cuenta los parámetros que son utilizados actualmente. En la Tabla 2 se muestra el rango en que debe encontrarse la resistencia para considerar el producto dentro de las especificaciones.

TABLA 2

Rango de Resistencia para Resistencia del Fusible TCF90

\begin{tabular}{|c|c|c|c|c|c|}
\hline PN & DATE & Mín & MÁx & Nom & Notes \\
\hline TCF90 & 15 -sep-04 & 0.790 & 0.982 & 0.886 & \\
\hline
\end{tabular}

Una vez obtenidos los datos relevantes al rango de resistencia, se capturó la información proporcionada por la corrida de validación y se realizó un estudio de capacidad con 30 datos en el software Minitab para obtener los índices de capacidad relevantes y determinar con ello la capacidad que se tiene para la producción normal del fusible.

En la Tabla 3 se pueden observar los valores capturados y en la Figura 2 se muestran los resultados analizados con ayuda de Minitab 19.

Los índices obtenidos son $C p=1.00$ y $C p k=0.25$, lo que alerta sobre una deficiencia en el proceso, por lo cual se determinó contemplar mejoras en el mismo para evitar defectos potenciales de calidad.
TABLA 3

Resultados de Resistencia de Corrida Piloto de Validación FuENTE: LOS AUTORES

\begin{tabular}{|l|l|l|l|l|l|}
\hline \multicolumn{1}{|c|}{$\# \mathrm{M}$} & \multicolumn{1}{|c|}{$\mathrm{R} \mathrm{m} \Omega$} & \multicolumn{1}{c|}{$\# \mathrm{M}$} & \multicolumn{1}{c|}{$\mathrm{R} \mathrm{m} \Omega$} & \#M & \multicolumn{1}{c|}{$\mathrm{R} \mathrm{m} \Omega$} \\
\hline 1 & 0.940 & 11 & 0.940 & 21 & 1.000 \\
\hline 2 & 0.960 & 12 & 0.940 & 22 & 1.010 \\
\hline 3 & 0.920 & 13 & 0.930 & 23 & 0.970 \\
\hline 4 & 1.010 & 14 & 1.010 & 24 & 0.940 \\
\hline 5 & 0.940 & 15 & 0.993 & 25 & 0.920 \\
\hline 6 & 0.930 & 16 & 0.970 & 26 & 0.990 \\
\hline 7 & 0.950 & 17 & 0.930 & 27 & 0.900 \\
\hline 8 & 0.910 & 18 & 0.980 & 28 & 0.980 \\
\hline 9 & 0.980 & 19 & 0.970 & 29 & 0.930 \\
\hline 10 & 0.990 & 20 & 0.950 & 30 & 0.960 \\
\hline
\end{tabular}

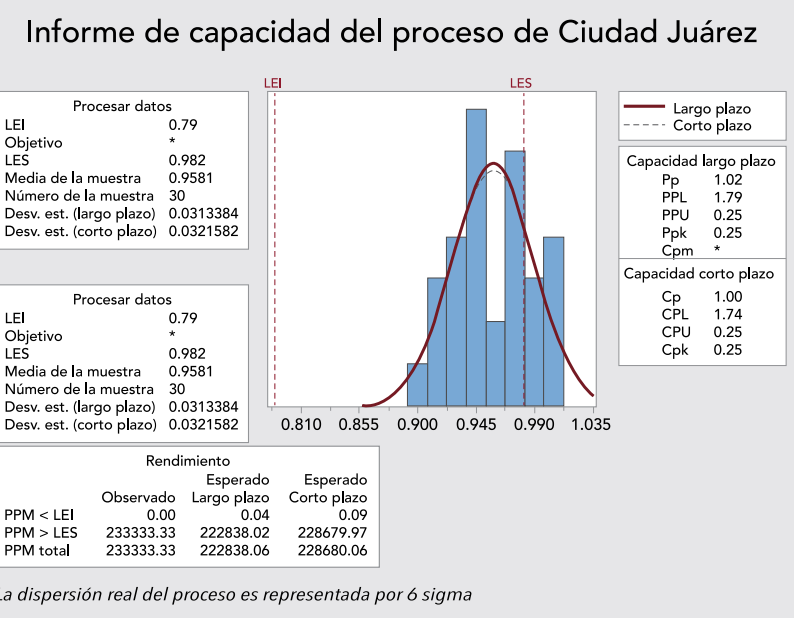

Figura 2. Índices de capacidad de la corrida piloto de validación. Fuente: los autores.

Los índices obtenidos son $C p=1.00$ y $C p k=0.25$, lo que alertó sobre una deficiencia en el proceso, por lo cual se contemplaron mejoras en el mismo para evitar defectos potenciales de calidad.

\section{Diseño factorial fraccionado}

En la corrida de validación piloto se manufacturó el fusible en la estación de soldado de trigger con los siguientes parámetros:

- Temperatura: $550^{\circ} \mathrm{F}$.

- Flujo de aire - A: 15 psi.

- Flujo de aire - B: 15 psi.

Lo anterior causó defectos de calidad, como lo son la soldadura escurrida y la falta de soldadura entre las uniones (bala-calentador y elemento-bala). 
Se consideró hacer un cambio en los tres parámetros para optimizar la calidad de las uniones. Los parámetros fueron los siguientes:

- Temperatura máx.: $610^{\circ} \mathrm{F}$.

- Temperatura mín.: $400^{\circ} \mathrm{F}$.

- Flujo A máx.: 27 psi.

- Flujo A mín.: 14 psi.

- Flujo B máx.: 30 psi.

- Flujo A mín.: 10 psi.

Para el diseño del experimento se realizó un DOE factorial fraccionado, con la finalidad encontrar los parámetros óptimos para la máquina y utilizar pocas muestras tomando rangos expandidos de temperatura y flujo.
El diseño se basó en un experimento factorial fraccionado $1 / 2$, de 2 niveles y 3 factores. Los factores que se consideraron son:

- Temperatura.

- Flujo-A.

- Flujo-B.

El diseño de experimentos fraccionado $2^{3-1}$ tiene 2 réplicas, lo que se tradujo en 8 corridas experimentales que se presentan en la Tabla 4. Además, contó con 2 puntos centrales, lo que sumó 2 corridas, dejando un total de 10 corridas para el diseño creado, además muestra las 4 variables de respuesta de interés, como se observa en la Tabla 4.

TABLA 4

Esquema de Experimentación y Resultados. Fuente: los Autores

\begin{tabular}{|c|c|c|c|c|c|c|c|c|c|c|}
\hline OrdenEst & OrdenCorrida & PtCentral & Bloques & \begin{tabular}{|l|} 
Flujo - \\
A (psi)
\end{tabular} & $\begin{array}{l}\text { Flujo - } \\
\text { B (psi) }\end{array}$ & Temperatura & Tiempo & $\begin{array}{c}\text { Soldado - Bala } \\
\text { / Calentador }\end{array}$ & $\begin{array}{c}\text { Escurrimiento } \\
\text { - Calentador }\end{array}$ & $\begin{array}{l}\text { Soldado - } \\
\text { Elemento }\end{array}$ \\
\hline 6 & 1 & 1 & 1 & 27 & 10 & 400 & 80 & 1 & 0.3 & 1 \\
\hline 1 & 2 & 1 & 1 & 14 & 10 & 610 & 61 & 1 & 0 & 0 \\
\hline 7 & 3 & 1 & 1 & 14 & 30 & 400 & 71 & 1 & 0 & 1 \\
\hline 4 & 4 & 1 & 1 & 27 & 30 & 610 & 33 & 1 & 0 & 0.5 \\
\hline 3 & 5 & 1 & 1 & 14 & 30 & 400 & 57 & 0.3 & 0 & 1 \\
\hline 10 & 6 & 0 & 1 & 20.5 & 20 & 505 & 48 & 0.5 & 0 & 0.8 \\
\hline 9 & 7 & 0 & 1 & 20.5 & 20 & 505 & 52 & 1 & 0.3 & 0 \\
\hline 8 & 8 & 1 & 1 & 27 & 30 & 610 & 30 & 0.5 & 0.2 & 1 \\
\hline 2 & 9 & 1 & 1 & 27 & 10 & 400 & 90 & 1 & 0.4 & 1 \\
\hline 5 & 10 & 1 & 1 & 14 & 10 & 610 & 47 & 1 & 0.4 & 1 \\
\hline
\end{tabular}

Al tomar los datos de las salidas, se aplicó el optimizador de respuesta del diseño de experimentos y se obtuvieron los diagramas de Pareto de efectos estandarizados para conocer cómo influyó cada factor en las salidas que se definieron como críticas, basados en la corrida piloto para obtener una mejor unión entre componentes, un menor escurrimiento de la soldadura en el calentador y el tiempo óptimo para el proceso.

A continuación, se muestran los diagramas de Pareto (Figuras 3, 4, 5 y 6) con una breve descripción del resultado que presentan.

En la Figura 3, el efecto mostrado respecto al tiempo es que a mayor temperatura se obtiene un menor tiempo para derretir la soldadura y unir los componentes del subensamble.
Figura 3. Diagrama de efecto estandarizado (La respuesta es Tiempo). Fuente: los autores.

La Figura 4 describe qué flujo tiene mayor efecto al soldar la bala con el calentador, por lo que indica que al tener un flujo alto se tiene una unión uniforme. 


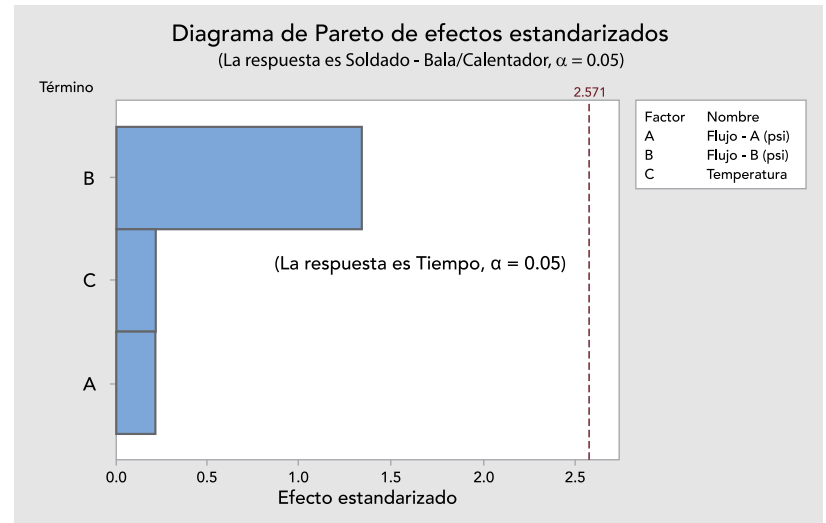

Figura 4. Diagrama de Pareto de efecto estandarizado (La respuesta es Soldado-Bala/Calentador). Fuente: los autores.

El diagrama de Pareto (Figura 5) muestra que a mayor flujo en el cautín de abajo existen más probabilidades de que haya escurrimiento.

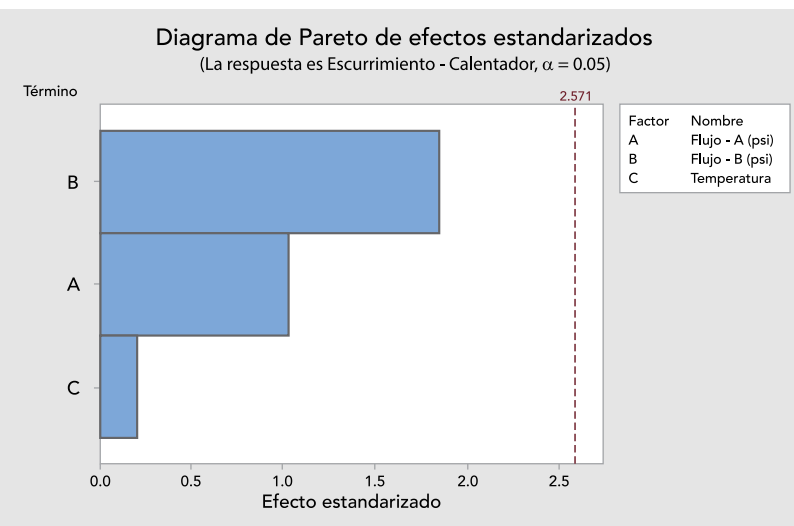

Figura 5. Diagrama de Pareto de efecto estandarizado (La respuesta es Escurrimiento-Calentador). Fuente: los autores.

En la Figura 6, el diagrama de Pareto muestra que la temperatura influye más en el soldado que el flujo.

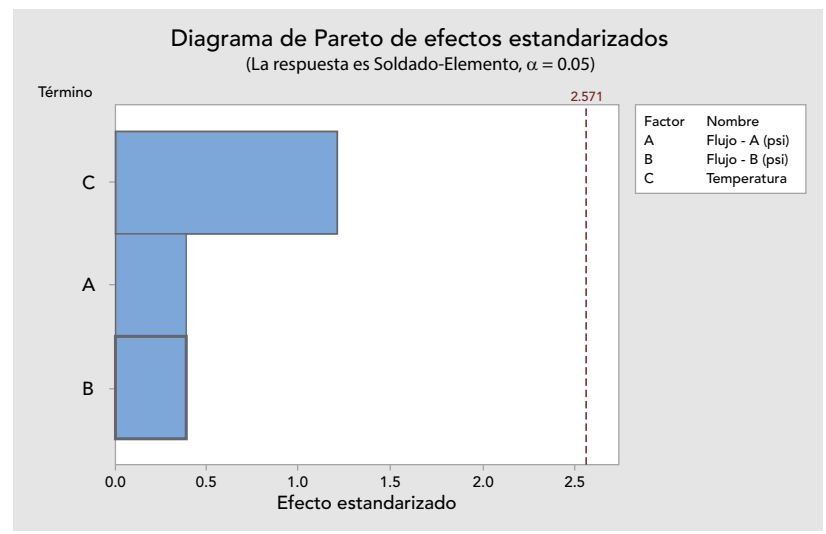

Figura 6. Diagrama de efecto estandarizado (La respuesta es Soldado-Elemento). Fuente: los autores.
Las salidas que se toman en cuenta para el diseño son:

- Tiempo. Para conocer cuánto tiempo se requería para la unión de los componentes.

- Soldado-Bala/Calentador. Se tomó el valor 1 como la mejor unión entre los componentes y 0 como la peor.

- Escurrimiento-Calentador. Se tomó el valor 0 como el mejor valor y conforme este aumenta significa que hay un escurrimiento mayor.

- Soldado-Elemento. Se tomó el valor 1 como la mejor unión del elemento y 0 como el peor.

Como resultado del análisis del diseño previamente realizado y analizado en Minitab, se obtuvo el diagrama de la Figura 7, en el que se puede observar las interacciones de las cuatro variables de respuesta con los tres factores que se definieron para el diseño experimental.

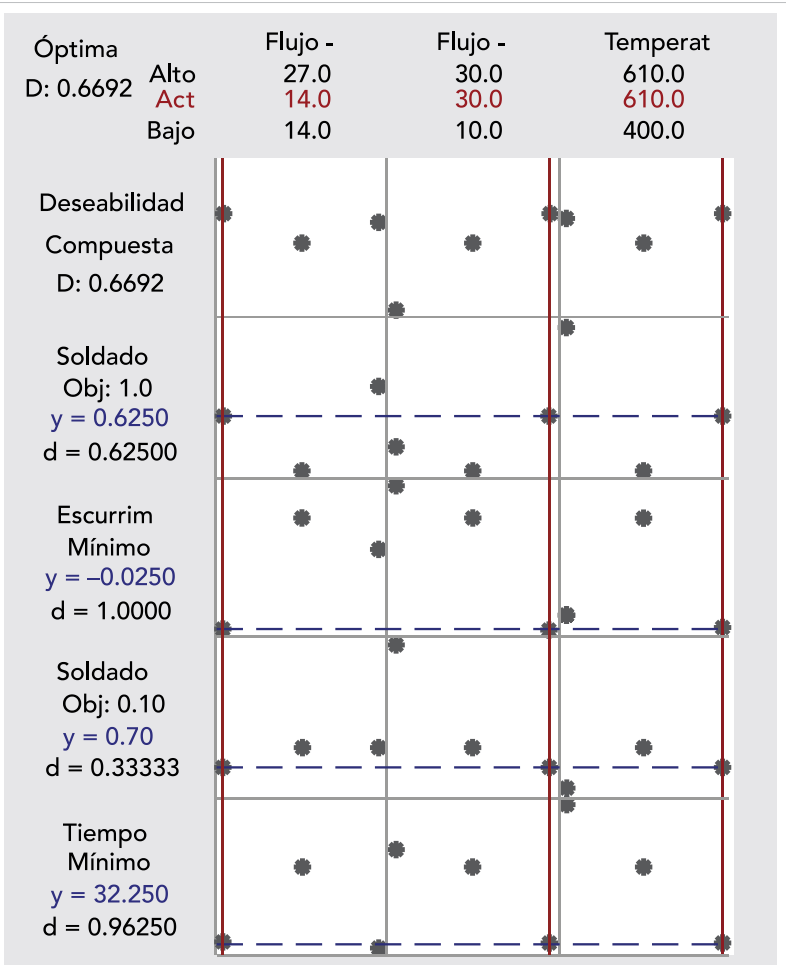

Figura 7. Diagrama de interacciones del diseño experimental. Fuente: los autores.

Al aplicar el optimizador de respuesta, se consideró un objetivo para la unión de bala y calentador; para el escurrimiento, se buscó el valor mínimo; para la unión bala y elemento, se buscó el mínimo; y el tiempo de proceso, se buscó el menor tiempo, dando como resultado que los parámetros óptimos para conseguir los objetivos planteados sean: 
- Flujo $\mathrm{A}=14$ psi.

- Flujo B $=30$ psi.

- Temperatura $=610^{\circ} \mathrm{F}$.

Por lo tanto, en la corrida de validación se deben hacer los cambios en los parámetros planteados y verificar el rango de resistencia.

\section{RESULTADOS Y DISCUSIÓN}

Con el fin de verificar los nuevos parámetros óptimos sugeridos por el diseño de experimentos, se hizo una corrida de validación con el fusible TCF90 de una muestra de 30 piezas, para obtener los datos de rango de frecuencia y posteriormente realizar el estudio de capacidad y obtener los índices de capacidad.

Se configuró la temperatura en el panel de la estación de soldado de trigger, como se muestra en la Figura 8. Enseguida se modificó el flujo de los cautines superior e inferior 14 psi y 30 psi respectivamente (Figura 9).

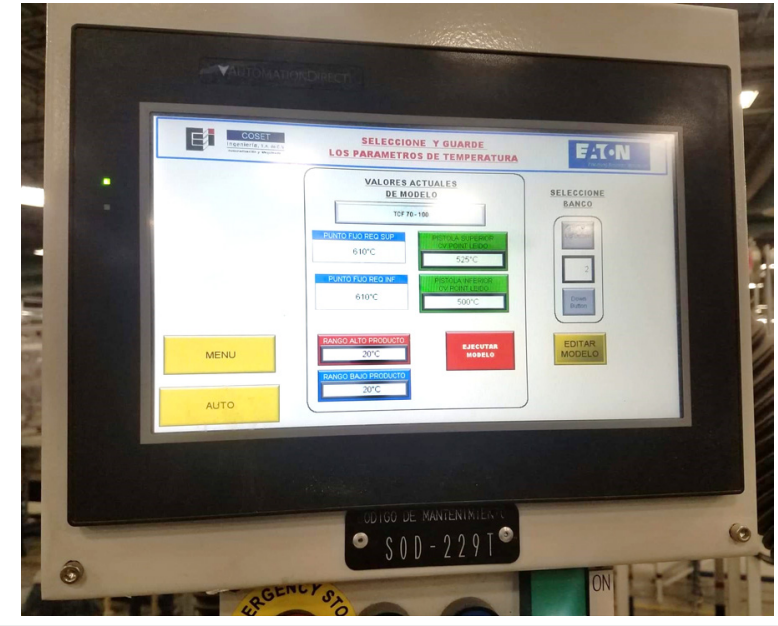

Figura 8. Pantalla de configuración para la Temperatura ${ }^{\circ} \mathrm{F}$. Fuente: los autores.

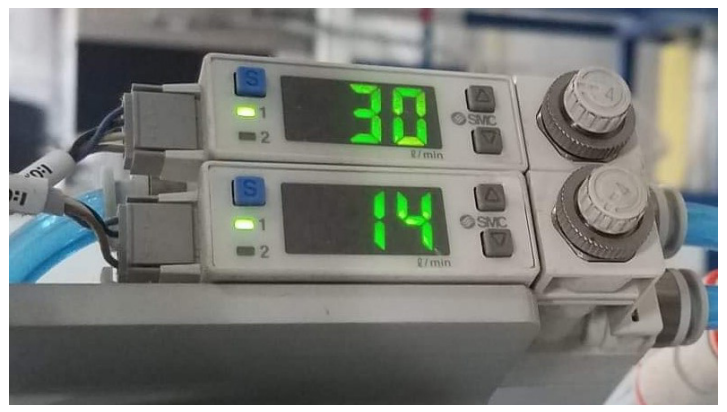

Figura 9. Perillas de control de flujo para cautines superior e inferior. Fuente: los autores.
Los datos obtenidos, después de la corrida de validación, se muestran en la Tabla 5.

TABLA 5

Valores de Resistencia de Corrida de Validación Experimental. Fuente: los Autores

\begin{tabular}{|c|c|c|c|c|c|}
\hline$\# \mathrm{M}$ & $\mathrm{R} \mathrm{m} \Omega$ & $\# \mathrm{M}$ & $\mathrm{Re} \mathrm{m} \Omega$ & $\# \mathrm{M}$ & $\mathrm{R} \mathrm{m} \Omega$ \\
\hline 1 & 0.921 & 11 & 0.952 & 21 & 0.962 \\
\hline 2 & 0.956 & 12 & 0.955 & 22 & 0.942 \\
\hline 3 & 0.943 & 13 & 0.942 & 23 & 0.948 \\
\hline 4 & 0.951 & 14 & 0.951 & 24 & 0.925 \\
\hline 5 & 0.943 & 15 & 0.945 & 25 & 0.934 \\
\hline 6 & 0.950 & 16 & 0.922 & 26 & 0.929 \\
\hline 7 & 0.952 & 17 & 0.943 & 27 & 0.943 \\
\hline 8 & 0.961 & 18 & 0.944 & 28 & 0.949 \\
\hline 9 & 0.943 & 19 & 0.935 & 29 & 0.945 \\
\hline 10 & 0.948 & 20 & 0.938 & 30 & 0.941 \\
\hline
\end{tabular}

Se analizaron los nuevos rangos de resistencia obtenidos previamente, con la finalidad de conocer el efecto real en el proceso, ya que se han realizado los cambios previstos con anterioridad. Además, con el estudio de capacidad se conoce si se han alcanzado los objetivos planteados al principio de la investigación.

Los índices de capacidad se muestran en la Figura 10.

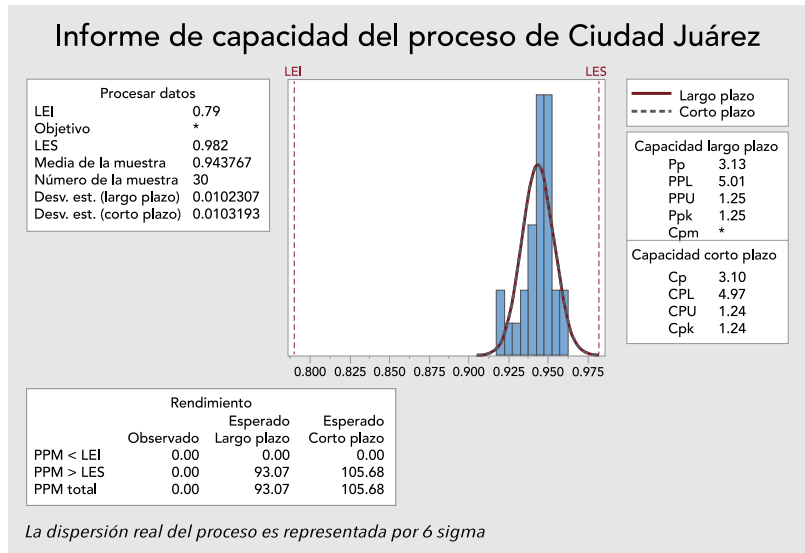

Figura 10. Informe de capacidad del proceso después de aplicar el diseño de experimentos. Fuente: los autores.

Los nuevos índices de capacidad son $C p=3.10$ y $C p k=$ 1.24. De acuerdo a la Tabla 1, se puede afirmar que se tiene un proceso de clase mundial, calidad seis sigma, y según [1], se tiene un proceso satisfactorio debido al valor de Cpk obtenido, por lo que se considera que se han cumplido los objetivos de la investigación. Como se puede notar en la Figura 10, la variación de los datos 
es muy pequeña, lo cual denota que se logró que el proceso genere productos con características estables. Por otra parte, la mejora en cuanto a capacidad del proceso se puede también notar en las partes por millón (ppm). Antes de la mejora, los ppm observados fueron 233333 mientras que después de la mejora quedaron en 105.68. Por supuesto, este es un impacto importante, dado que el número de piezas malas por millón se redujo drásticamente.

Se puede realizar una comparación del proceso antes y después del diseño de experimentos de manera gráfica, como se muestra en la Figura 11.

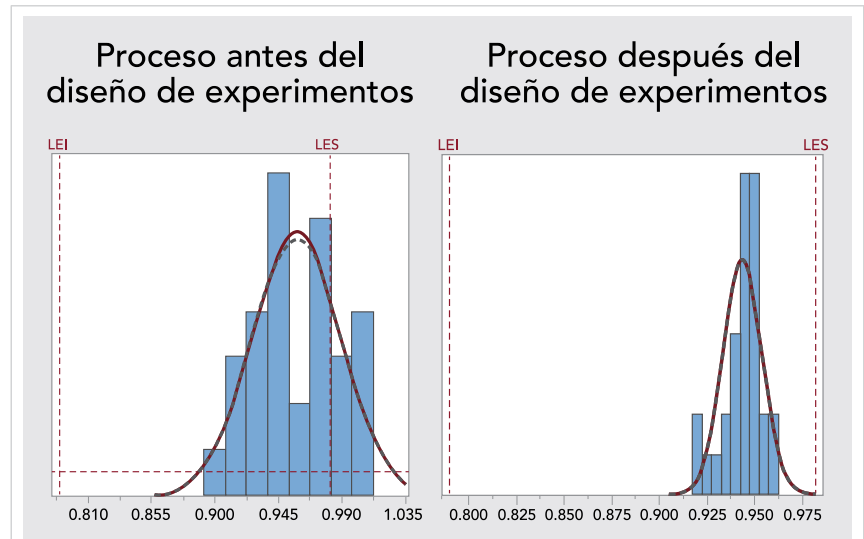

Figura 11. Comparación de gráficas de capacidad del proceso. Fuente: los autores.

Se puede observar cómo los datos muestran una mejora respecto a la distribución y la variabilidad, así como en los índices de capacidad $C p$ y $C p k$, obteniendo resultados positivos en cuanto a lo requerido por las especificaciones del cliente.

Específicamente, se puede notar que se logró reducir la variación de los datos después del diseño de experimentos. Esto se puede advertir visualmente con el histograma de menor amplitud del lado derecho de la Figura 11. De igual manera, se puede notar que la distribución de los datos está dentro de los límites de especificación y un poco más centrada en comparación del histograma del proceso antes del diseño de experimentos. Ambas cuestiones, centrado y reducción de variación, son características importantes que se toman en cuenta tanto en el $C p k$ con el $C p$.

Como se puede notar en las Ecuaciones (1) y (3), lo centrado del proceso se evalúa conforme al numerador de (3) y la amplitud de variación conforme al denominador de (1). Por esta razón se obtuvieron mejores resultados en el proceso después de llevar el esquema de experimentación.

\section{CONCLUSIONES}

El proyecto tiene como objetivo general optimizar la estabilidad del proceso para el fusible TCF90 respecto a la resistencia. Para lograrlo, se requiere del uso de herramientas estadísticas para analizar los datos con base en los índices de capacidad de proceso, determinar la estabilidad del mismo y el diseño de experimentos, buscar una optimización de las variables de interés y encontrar la mejor unión entre elementos que componen el fusible.

Para demostrar esto, se hizo una corrida de validación piloto con la cual se encontró que el proceso tiene deficiencias respecto a los estándares de calidad, lo cual alertó sobre defectos potenciales en el producto que se manufactura.

Los defectos que se encontraron sugieren que el proceso de soldado es la causa principal, por lo que el diseño de experimentos realizado se enfocó en la parte en la cual se crea la unión entre los elementos del fusible. Debido a los recursos con los que se contaban para la validación del producto, se tomó la decisión de crear un diseño factorial fraccionado para consumir menor cantidad de insumos, ya que este solo implica una parte del diseño y no el total de las réplicas del diseño factorial completo.

Con la implementación de los parámetros sugeridos por el diseño de experimentos, se observó una mejora en el soldado de los elementos para el fusible en cuestión, lo que indica que el diseño es funcional para el propósito planteado.

Este cambio en los parámetros de temperatura y flujo para el proceso dio como resultado un impacto en los índices de capacidad, volviendo estable al proceso y con índices de acuerdo a los estándares de calidad. Después de todo, las empresas buscan mantener la eficiencia en sus procesos para evitar desperdicios de material y quejas de cliente, por lo que se puede determinar que de manera general se cumplió con el objetivo, logrando mayor estabilidad en el proceso de manufactura del fusible. 


\section{REFERENCIAS}

[1] H. Gutiérrez y R. de la Vara Salazar, Control estadístico de la calidad y seis sigma, 3. ${ }^{\mathrm{a}}$ ed. México, D.F.: McGraw Hill Education, 2013.

[2] A. Patricia, R. Picón, L. A. R. Picón, M. A. R. Medina, y S. R. Picón, "Índice de capacidad para una tolerancia posicional: Caso de estudio", Cultura Científica y Tecnológica, año 13, no. 59, pp. 365-374, 2016.

[3] J. R. Evans y W. M. Lindsay, Administración y Control de Calidad, 9. ${ }^{\mathrm{a}}$ ed. México, D.F.: Cengage Learning Editores, 2014.

[4] H. Gutiérrez y R. De la Vara, Análisis y diseño de experimentos, 3. a ed. México: McGraw Hill, 2012.

[5] C. Hernández y F. Da Silva, "Aplicación del control estadístico de procesos (CEP) en el control de su calidad", Tecnología química, vol. 36. no. 1, pp. 104-116, 2016. Disponible en: http://scielo.sld.cu/pdf/rtq/v36n1/ rtq10116.pdf

[6] C. A. Doncel y D. E. Marín, "Índices de capacidad, producto fuera de especificación y pérdidas asociados al proceso de llenado de frascos en una empresa del área cosmética", Fundación Universitaria Los Libertadores, Facultad de Ingeniería y Ciencias Básicas, Especialización en Estadística Aplicada, Bogotá D.C., Colombia, 2019. Consultado en 2020. [En línea]. Disponible en: https://repository.libertadores.edu.co/ handle/11371/2790

[7] C. Huerga, J. I. Abad y P. Blanco, "El papel de la Estadística en la metodología Seis Sigma: una propuesta de actuación en servicios sanitarios", Pecvnia : Revista de la Facultad de Ciencias Económicas y Empresariales, no. 2012, pp. 111-136, mar. 2012. DOI: 10.18002/pec. v0i2012.1109.

[8] C. Acheson, D. Mackle, A. Murphy, J. Butterfield, P. Higgins, R. Collins, C. Higgins, J. Darlington y R. Tame, Using Design Of Experiments To Define Factory Simulations For Manufacturing Investment Decisions. Belfast: Queen's University Belfast, 2017, pp. 1-9 [En línea]. Disponible en: https://pureadmin.qub.ac.uk/ws/portalfiles/portal/134852763/IMC34_ACHESON_Final.pdf. [Consultado en 2020]

[9] B. C. Gupta, I. Guttman y K. P. Jayalath, Statistics and Probability with Applications for Engineers and Scientists using MINITAB, $R$ and JMP, 2. ${ }^{\mathrm{a}}$ ed. Hoboken, Nueva Jersey: John Wiley \& Sons, Inc., 2020.

[10] G. P. Ortiz, C. Anaya, G. Ortiz y P. D. Sánchez, Aplicación de un diseño de Experimentos $2^{k}$ para la Optimización de un Proceso de Moldeo por Inyección", Hermosillo, Sonora: Universidad de Sonora, 2014, pp. 11-20 [En línea]. Disponible en: http://www.irsitio.com/refbase/ documentos/175_OrtizEspinoza_etal2014.pdf. [Consultado en 2020]

[11] A. Díaz, Diseño estadístico de experimentos, 2. ${ }^{\text {a }}$ ed. Medellín, Colombia: Universidad de Antioquía, 2009.

[12] O. O. Melo, L. A. López y S. E. Melo, Diseño de Experimentos [Métodos y Aplicaciones], Bogotá, Colombia: Universidad Nacional de Colombia, 2003.

[13] J. Lawson, Design and Analysis of Experiments with $R$, 1. a ed. Boca Raton, Florida: CRC Press, 2015. 